\title{
Synthesis of Mesopores of Zirconia by Using CTAC as Template
}

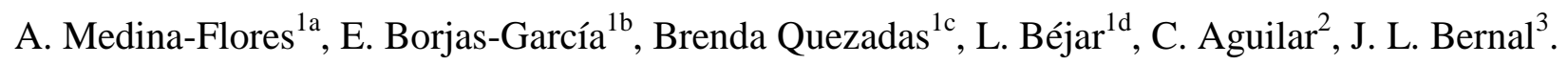

${ }^{1 \mathrm{a}}$ Instituto de Investigaciones Metalúrgicas, ${ }^{1 \mathrm{~b}}$ Instituto de Física y Matemáticas, ${ }^{1 \mathrm{c}}$ Facultad de Ciencias Físico y Matemáticas, ${ }^{1 \mathrm{~d}}$ Facultad de Ingeniería Mecánica, Universidad Michoacana de San Nicolás de Hidalgo, Morelia, Michoacán, México, C.P. 58000

${ }^{2}$ Depertamento de Ingeniería Metalúrgica y Materiales. Universidad Técnica Federico Santa María.

Av. España 1680, Valparaíso, Chile.

${ }^{3}$ Automotive Mechanics Department. Universidad Politécnica de Pachuca. Zempoala, Hidalgo. México

A mesoporous material is a material, which contains pores in a range between 2 and 50 nanometers [1]. This type of material can be used as catalyst for bulky reactant molecules due their large pore size. The first publication about synthesis of mesoporous materials by using a surfactant template was in 1992 by Mobil scientists [2]. After that, several methods have been developed for the synthesis of mesoporous metal oxides [1]. However, the synthesis of mesoporous zirconia by using Cetyltrimetylammonium chloride (CTAC) as template has not investigated. The mesopores of zirconia was prepared by using zirconium oxide chloride octahydrate (Sigma-Aldrich, purity 99.5\%), tetramethylammonium hydroxide solution TMAOH (Sigma-Aldrich, $25 \mathrm{wt} \%$ in water) and cetyltrimethylammonium chloride solution, CTAC (Aldrich, $25 \mathrm{wt} \%$ in water) as source. In a first step, $1.611 \mathrm{~g}$ of $\mathrm{ZrOCl}_{2} * 8 \mathrm{H}_{2} \mathrm{O}$ was dissolved in $7.2 \mathrm{~g}$ of destilled water. Then, $3.2 \mathrm{~g}$ of CTAC solution was added to the $\mathrm{Zr}$-solution. For the second step, $10.94 \mathrm{~g}$ of TMAOH solution was added slowly (drop by drop) to Zr-surfactant solution and stirred. The final suspension was stirred and heat in a hot stir plate at $90{ }^{\circ} \mathrm{C}$ to get a material with a molar ratio of $\mathrm{ZrOCl}_{2} * 8 \mathrm{H}_{2} \mathrm{O}: \mathrm{CTAB}: \mathrm{TMAOH}: \mathrm{H}_{2} \mathrm{O}$ equal to $1: 0.5: 6: 75$. The gel obtained was aged in a polypropylene bottle at $80{ }^{\circ} \mathrm{C}$ for 1 day. After that, the hydrothermal treatment, the sample was washed with distilled water, centrifuged and dried at $80{ }^{\circ} \mathrm{C}$ for 1 day. The powder X-ray diffraction patterns were collected with a siemens D5000 X-ray diffractometer equipped with graphite monochromatized high-intensity $\mathrm{CuK}_{\lambda}\left(\lambda=1.54178 \AA\right.$ ). The Bragg angle $2 \theta$ ranges from $1^{\circ}$ to $8^{\circ}$ at a scanning rate of $0.02^{\circ} / \mathrm{s}$. The surface morphology images of the samples were analyzed by using a scanning electron microscopy FEG-SEM JEOL JSM 7600. Figure 1 shows the XRD pattern of zirconia synthesized. This figure shows a characteristic peak about the presence of mesopores in the material around 1.04 degrees. Figure 2a shows an image of mesoporous zirconia. Figure $2 b$ shows the EDS spectrum of the mesopores. The results shows that the optimal hydrothermal treatment temperature for mesoporous zirconia synthesis is at $80{ }^{\circ} \mathrm{C}$ because more temperature of treatment could collapse mesopores formation in zirconia and it was developed an easy procedure for the synthesis, which can be, applied at different materials.

\section{References}

[1] D.W. Bruce, et al., Volume 1 (2010), p. 1.

[2] C. T. Kresge et al, Nature Volume 359 (1992) p. 710.

[3] The authors acknowledge funding from H. Consejo Técnico of Institute of Physics and Mathematics, and Consejo Nacional de Ciencia y Tecnología (CONACyT), México. 


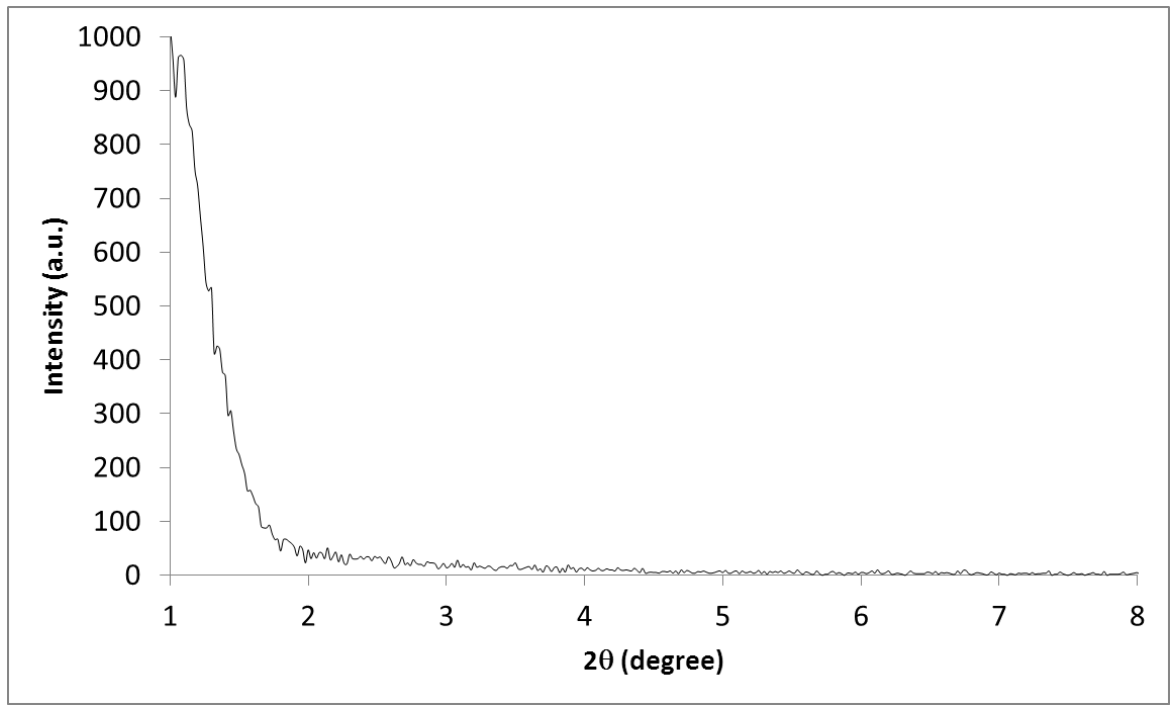

Figure 1. XRD pattern of mesoporous zirconia
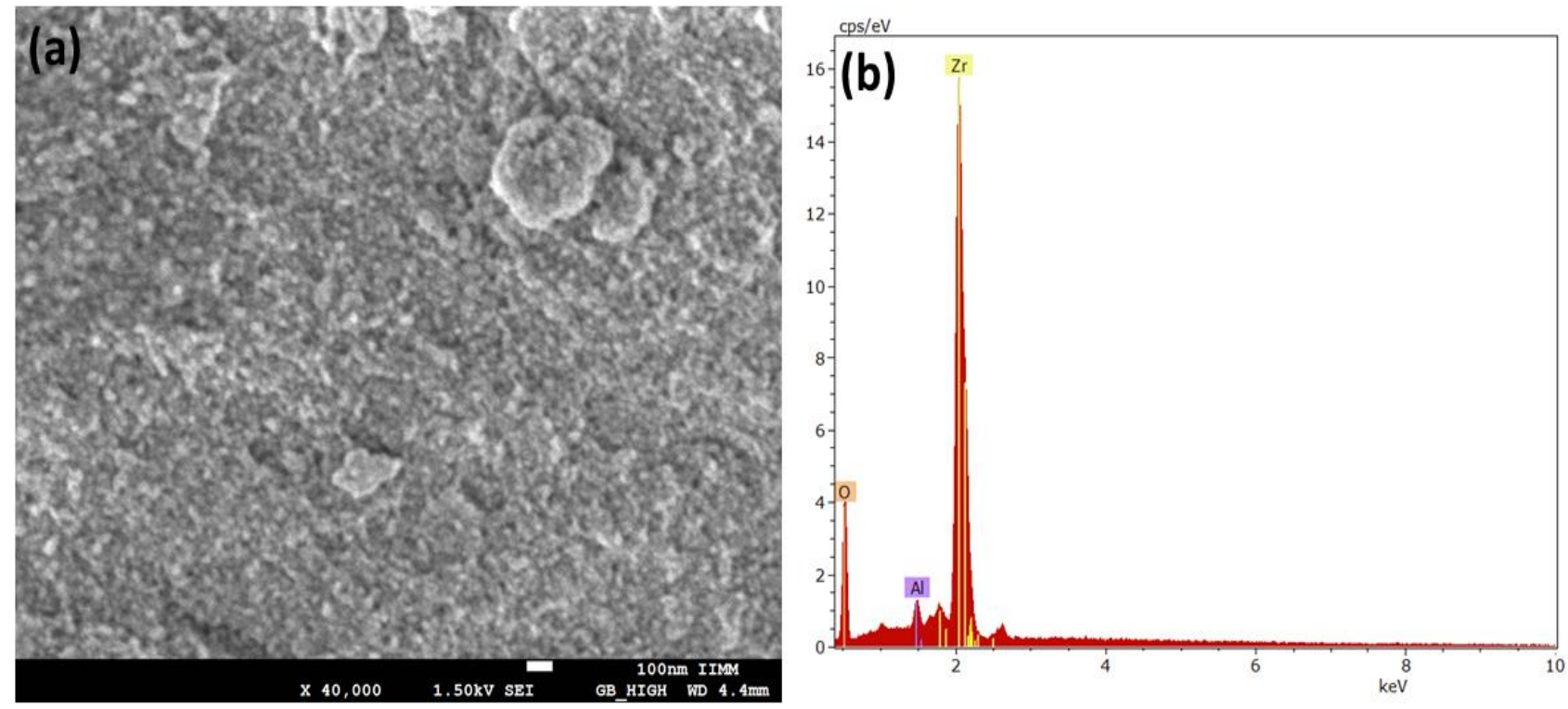

Figure 2. a) SEM image of mesoporous zirconia, b) EDS spectrum of the mesoporous of zirconia. 